\title{
Microwave mutagenesis of high-producing rennet strain from Bacillus subtilis
}

\author{
Mei Lin ${ }^{1,2}$, Li Wang ${ }^{2}$, Qing-qing Cao ${ }^{2}$ and Guang-Hong Zhou ${ }^{1 *}$ \\ ${ }^{1}$ Key Lab of Meat Processing and Quality Control, College of Food Science and Technology, Nanjing Agricultural \\ University, Nanjing, 210095, PR China. \\ ${ }^{2}$ College of Tea and Food Technology, Anhui Agricultural University, Hefei, 230036, PR China.
}

Received 28 March, 2013; Accepted 13 January, 2014

\begin{abstract}
In order to acquire a high-producing rennet strain from Bacillus, different microwave irradiation intensities and durations were tested. The effect of microwave irradiation power and time were studied and the microwave mutagenesis parameters were optimized. The results indicated that the best conditions for microwave mutagenesis that produced better performed mutant strains were $450 \mathrm{~W}, 4$ min. Under these conditions a viable strain with high-producing rennet (Strain No.: BsB1.1) was achieved whose genetic stability is good and rennet activity reaches $516.12 \mathrm{U} / \mathrm{g}$ which has increased by $\mathbf{2 9 . 0 \%}$ compared with the original strain in the experiment.
\end{abstract}

Key words: Bacillus subtilis, high-producing rennet, microwave mutagenesis.

\section{INTRODUCTION}

Rennet is the zymin used for milk or goat milk solidification in the cheese making and it is the crucial enzyme for milk solidification in cheese production. As the main additive, rennet activity has a greater influence on the quality of cheese products and will determine the texture and tastes of the products.

The current method used to obtain chymosin mainly involves three types: the first is extraction of enzyme from the stomachs of unweaned calves. This process is expensive and the supply is subject to the fluctuations in the veal market. The second is from plants, but its production is restricted by time, geographical conditions and so on, therefore it is also not suitable for mass production. The third is from microorganism. Rennet produced by microorganism is not only in low costs but its productivity can be expanded unlimitedly, so microorganism milk-clotting enzyme is one of the most promising development directions. However, the rennetproducing capability was relatively weak and therefore lacked of positive utilization value.

Mutagenesis could overcome the disadvantage of weak rennet-producing capability of wild-type strains. Microwave irradiation induces polar molecules around the cell walls to produce intense electric polarity oscillation (Kirsschvink, 1996; Chen et al., 2006), damages hydrogen bonds and base stacking forces, causes changes to DNA structure as well as the damages on the surface of microbial cells (Woo et al., 2000), and therefore results in chromosome changes and the production of mutants with desirable variation of genetic

\footnotetext{
*Corresponding author. E-mail: ghzhou@njau.edu.cn. Tel: 13905160737.
}

Author(s) agree that this article remain permanently open access under the terms of the Creative Commons Attribution License 4.0 International License 
characters (Hong et al. 2004; Li et al. 2009a,b; Pan et al. 2008; Selvakumar et al. 2008; Lamsa et al. 1990). Compared with other mutational approaches (for instance: chemical mutagenesis and UV light irradiation), the advantages of microwave irradiation include the avoidance of toxic substance production, the convenience of operation and the potential obtaining of numerous mutants in short time (Xu et al., 2009). For instance, Song et al. (2008) observed higher mutation rate and obtained anaerobic hydrogen producing strain $\mathrm{H}-8$, hydrogen production of the obtained variant HW195 was $50.7 \%$ higher than that of the original strain. Xu et al. (2009) also obtained high yields of ideal strains L-lactic acid bacteria by microwave mutagenesis. Nonetheless, no practical application of microwave mutagenesis for Bacillus subtilis has been reported. This study has the following aims: to obtain mutant strains of $B$. subtilis BsB1.1 with high rennet producing capability by microwave mutagenesis, to establish optimum mutagenesis conditions, and to produce reference materials for the study of rennet with efficient rennet -producing capability.

\section{MATERIALS AND METHODS}

\section{Bacterial strain}

The wild-type rennet -producing $B$. subtilis BsB1.1 isolated from the cheese of Co. YILI of the People's Republic of China. The strain BsB1.1 had been identified as $B$. subtilis based on biochemical tests, and a 1.5-kbp sequence of the 16S rRNA gene region which was fully determined and analyzed by the Clustal W program (Gen Bank accession: KC492497). The strain BsB1.1 was able to grow in Casein media and was found possessing rennet -producing capability. Pure cultures were maintained in casein agar (CA) slants at $4^{\circ} \mathrm{C}$ until needed.

\section{Medium}

\section{Casein medium}

Preparation of crude enzyme: Inoculate bacteria fluid at $2 \%$ proportion on casein fluid nutrient medium at $30^{\circ} \mathrm{C}$, shake cultivation for 2 days at $180 \mathrm{r} / \mathrm{min}$, and then put all the fluid into centrifugal tube, centrifuge for $30 \mathrm{~min}$ at $3,500 \mathrm{rpm}$. And the supernatant in the tube is crude enzyme.

\section{Determination of rennet activity}

Rennet activity was determined according to Arima method. Take 5 $\mathrm{mL} 100 \mathrm{~g} / \mathrm{L}$ skim milk, preserve heat for $5 \mathrm{~min}$ at $35^{\circ} \mathrm{C}$ in water bath, and then add $0.5 \mathrm{~mL}$ fermentation broth, mix them in the vortex mixer and record accurately the time from enzyme adding to milk solidification.

Sohxlet Unit (SU): The enzyme number in 40 min solidifying $1 \mathrm{~mL}$ $100 \mathrm{~g} / \mathrm{L}$ skim milk.

$S U=(2400 \times 5 \times D) /(T \times 0.5)$

Where, $T$ represents the coagulating time, and $D$ represents the dilution multiple of the enzyme.

\section{Determination of proteolysis test}

Five milliliters $1.2 \%$ casein solution was added to $1 \mathrm{~mL}$ enzyme that has been diluted tenfold, and heat preserved for $10 \mathrm{~min}$ at $35^{\circ} \mathrm{C}$. Enzyme reaction was terminated with $5 \mathrm{~mL} 0.44 \mathrm{~mol} / \mathrm{L}$ TCA, and heat continually preserved for 20 min. Reaction mixture was filtered with filter paper. Two milliliters filtrate was taken and $5 \mathrm{~mL} 0.55$ $\mathrm{moL} / \mathrm{INa}_{2} \mathrm{CO}_{3}$ solution and $1 \mathrm{~mL} 0.7 \mathrm{~mol} / \mathrm{L}$ Folin reagent added. Heat was preserved for $20 \mathrm{~min}$ at $35^{\circ} \mathrm{C}$, and absorbance measured at $660 \mathrm{~nm}$ wavelength.

Proteolysis activity $=(\mathrm{A} \times \mathrm{K} \times \mathrm{V}) /(\mathrm{N} \times \mathrm{t})$

Where: A represents the absorbance at $660 \mathrm{~nm}$ wavelength; $\mathrm{K}$ represents the tyrosine micrograms/Mg when light absorption is " 1 " in the standard curve; $\mathrm{V}$ represents the total volume $/ \mathrm{mL}$ of enzymatic reaction; $t$ represents the time/min of enzymatic reaction; $\mathrm{N}$ represents the dilution multiple of the enzyme.

\section{Screening of original strain}

Preserved strain from the plate in the laboratory was selected and coagulating effect observed. Rennet activity and proteolysis activity of the strain was determined. The strain that have favorable coagulating effect, high rennet activity and low proteolysis activity was original one.

\section{Screening of the best mutagenic time}

Ten milliliters $B$. subtilis suspension was taken into $50 \mathrm{~mL}$ triangular flasks. The triangular flasks were sealed with sealing film, and the triangular flasks placed into a container filled with ice, and then the container put in a microwave for treatment. The mutagenic power was $450 \mathrm{~W}$, and the mutagenic time 2, 3, 4, 5 and $6 \mathrm{~min}$, respectively. After irradiation, the triangular flasks were moved on a clean bench; and $100 \mathrm{ul}$ suspension taken from each triangular flask, respectively for dilution to $10^{-4}, 10^{-5}$, and $10^{-6}$ times. They were coated on the casein culture plate after refrigeration and protection from light for $3 \mathrm{~h}$. The $B$. subtilis suspension was coated before treatment under the same conditions, and protected from light for cultivation for 2 days. And then the clump counts were added up. The death rate, the positive and negative mutation rate was calculated and the best mutagenic time determined. Each experiment was done for 3 times.

\section{Screening of the best mutagenic power}

Ten milliliters of $B$. subtilis suspension was taken into $50 \mathrm{~mL}$ triangular flasks. The triangular flasks were sealed with sealing film, and the triangular flasks placed into a container filled with ice, and then the container put into a microwave for treatment. The mutagenic time was $4 \mathrm{~min}$, and the mutagenic power 350, 400, 450 and $500 \mathrm{~W}$, respectively. After irradiation, the triangular flasks were moved on a clean bench; and $100 \mathrm{ul}$ suspension taken from each triangular flask respectively for dilution to $10^{-4}, 10^{-5}$ and $10^{-6}$ times. They were coated on the casein culture plate after refrigeration and protection from light for $3 \mathrm{~h}$. The $B$. subtilis suspension was coat before treatment under the same conditions, and protected from light for cultivation for 2 days. And then the clump counts were added up. The death rate, the positive and negative mutation rate was calculated and the best mutagenic time determined. Each experiment was done for 3 times. 
Table 1. Screening results of three Bacillus Subtilis preserved in the laboratory

\begin{tabular}{|c|c|c|c|}
\hline Strain No. & curd effect & $\begin{array}{l}\text { Chymosin } \\
\text { activity (U/g) }\end{array}$ & $\begin{array}{l}\text { Proteolysis } \\
\text { activity (U/g) }\end{array}$ \\
\hline BsB1 & $\begin{array}{l}\text { Better flavor, tight and smooth texture, moderate hardness with the color of } \\
\text { cream yellow and even gloss }\end{array}$ & 399.96 & 1.280 \\
\hline BsB2 & $\begin{array}{l}\text { Favorable flavor, basically even but slightly soft texture, relatively tight tissue } \\
\text { with a slight change of color and luster }\end{array}$ & 363.60 & 2.633 \\
\hline BsB3 & $\begin{array}{l}\text { Acceptable flavor, loose tissue with a slight change of color and luster and } \\
\text { fragility }\end{array}$ & 355.52 & 2.898 \\
\hline
\end{tabular}

\section{Screening of the mutagenic strain}

Mutagenic strains were selected with bigger curds circle and little bacteriostasis circle, coagulating effect of the mutagenic strains were observed, respectively and rennet activity, proteolysis activity and stability of the strains determined. The screened strain that has favorable coagulating effect, high rennet activity, low proteolysis activity and genetic stability were target strains.

Texture analysis of the cheese by rennet from original strain and objective strain

Crude rennet solution was prepared by the same way. Then the rennet form original and objective strain was used to make cheese separately to contrast the texture by texture analyzer.

\section{RESULTS AND ANALYSIS}

\section{Screening of original strains}

The results of screening original strains are shown in Table 1. BsB1 strain has a better flavor, tight and smooth texture, moderate hardness with the color of cream yellow and even gloss. Rennet activity of BsB1 strain is $399.96 \mathrm{U} / \mathrm{g}$, and its proteolysis activity is $1.280 \mathrm{U} / \mathrm{g}$. BsB2 strain has a favorable milk-clotting enzyme flavor, basically even but slightly soft texture, relatively tight tissue with a slight change of color and luster. Rennet activity of BsB2 strain is $363.60 \mathrm{U} / \mathrm{g}$, and its proteolysis activity is $2.633 \mathrm{U} / \mathrm{g}$. BsB3 strain has the characteristics of an acceptable flavor, loose tissue with a slight change of color and luster, and fragility. Rennet activity of BsB3 strain is $355.52 \mathrm{U} / \mathrm{g}$, and its proteolysis activity is 2.898 $\mathrm{U} / \mathrm{g}$. BsB1 strain has the best coagulating effect, maximum rennet activity and minimum proteolysis activity. Therefore, BsB1 strain is chosen as the original strain of this experiment.

\section{Effects of mutagenic time on death rate and positive mutation rate}

With the extension of the mutagenic time, the death rate of the strain has increased to some extent (Figure 1). When the mutagenic time is $4 \mathrm{~min}$, the death rate of the suspension is $73 \%$. With the extension of the mutagenic time, the positive mutation rate increases. When the death rate of the suspension is $73 \%$, the positive mutation rate reaches the maximum. After that, with the increase of mutagenic power, the positive mutation rate has continuously decreased. Therefore, $4 \mathrm{~min}$ as the mutagenic time of microwave mutagens is chosen.

\section{Effect of mutagenic power on death rate and positive mutation rate}

The experimental result is shown in Figure 2. With the increase of the mutagenic power, the death rate of the strain has increased to some extent. When the mutagenic power is $450 \mathrm{~W}$, the death rate of the suspension is $76 \%$. With the increase of the mutagenic power, the positive mutation rate increases. When the death rate of the suspension is $76 \%$, the positive mutation rate reaches the maximum. After that, with the increase of mutagenic power, the positive mutation rate has a tendency to decrease. Therefore, choose $450 \mathrm{~W}$ as the mutagenic power of microwave mutagenesis.

\section{Screening of mutagenic strain}

From Table 2, coagulating texture of BsB1.1 strain is the tightest and the coagulating time is much more shortened; rennet enzyme activity of BsB1.1 strain is $516.12 \mathrm{U} / \mathrm{g}$, and its proteolysis activity is $1.296 \mathrm{U} / \mathrm{g}$. Coagulating texture of BsB1.2 strain is slightly tight and the coagulating time is more shortened; rennet activity of BsB1.2 strain is $457.20 \mathrm{U} / \mathrm{g}$, and its proteolysis activity is $2.530 \mathrm{U} / \mathrm{g}$. Coagulating texture of BsB1.3 strain is slightly tight and the coagulating time is slightly shortened; rennet activity of BsB1.3 strain is $417.36 \mathrm{U} / \mathrm{g}$, and its proteolysis activity is $2.784 \mathrm{U} / \mathrm{g}$. BsB1.1 strain has the best coagulating effect and its milk-clotting enzyme activity has increased most. So BsB1.1 strain is the target strain 


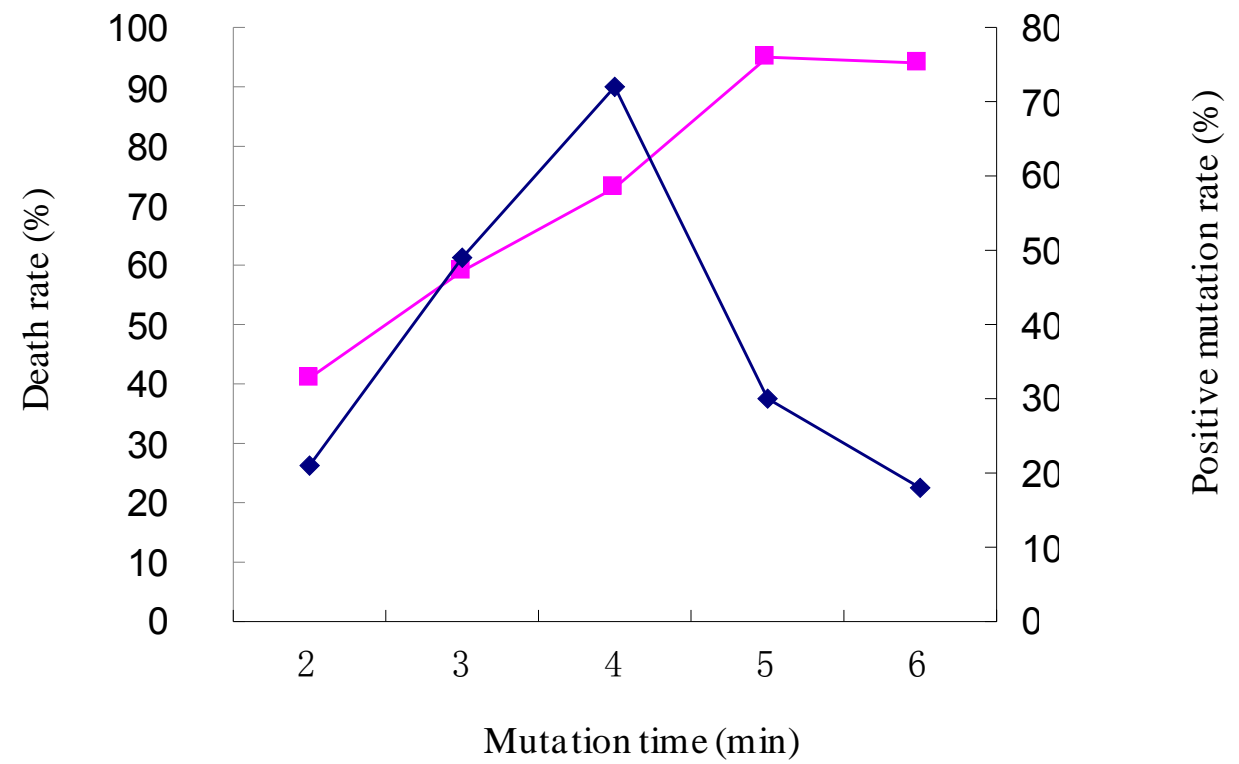

Figure 1. Effects of mutagenic time on death rate and positive mutation rate. $\square$ Death rate $(\%), \Delta$ Positive mutation rate (\%).

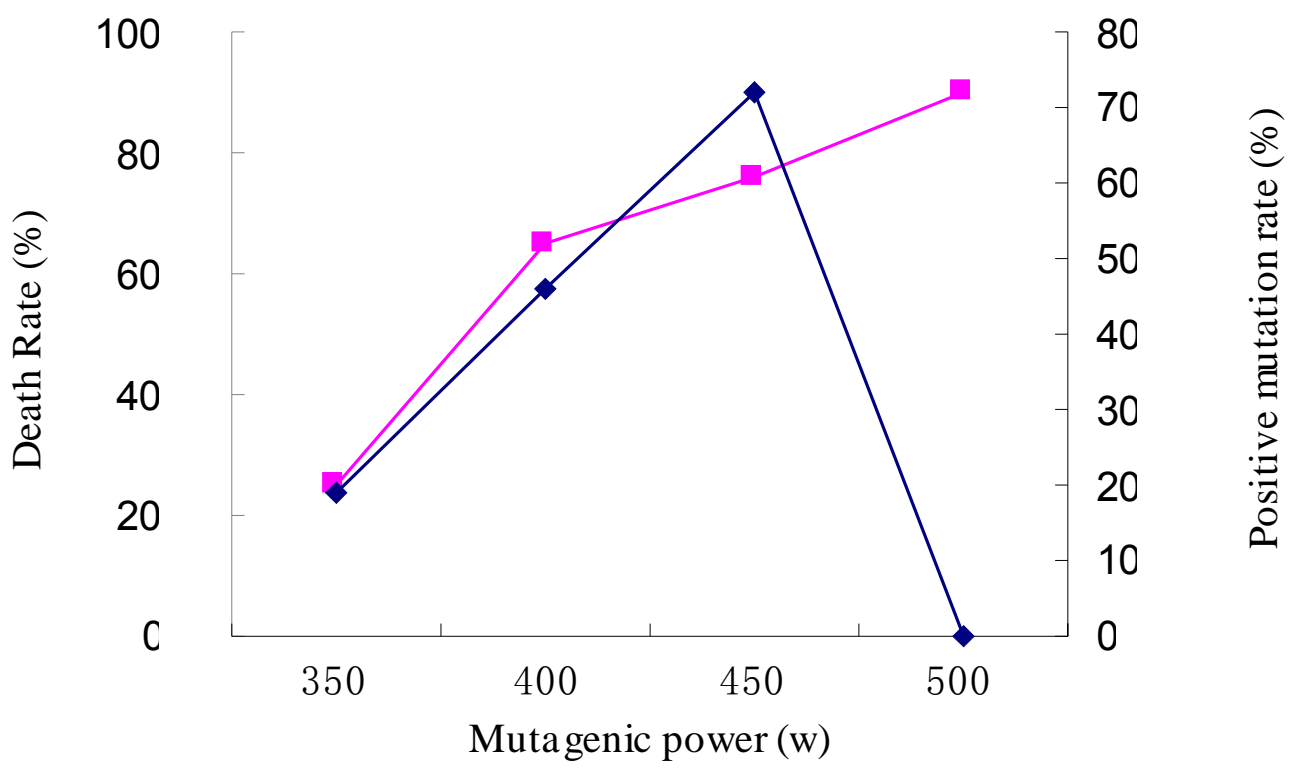

Figure 2. Effect of mutagenic power on death rate and positive mutation rate. $\square$ Death rate (\%); $\Delta$ Positive mutation rate (\%).

Table 2. Screening results of strains after microwave mutagenesis of BsB1

\begin{tabular}{llcc}
\hline Strain No. & Coagulating effect & $\begin{array}{c}\text { chymosin } \\
\text { activity (U/g) }\end{array}$ & $\begin{array}{c}\text { Proteolysis } \\
\text { activity (U/g) }\end{array}$ \\
\hline BsB1.1 & Tightest texture and coagulating time much more shortened & 516.12 & 1.296 \\
BsB1.2 & Slightly tight texture and coagulating time more shortened & 457.20 & 2.530 \\
BsB1.3 & Slightly tight texture and coagulating time slightly shortened & 417.36 & 2.784 \\
\hline
\end{tabular}


Table 3. Inspection results of stability.

\begin{tabular}{lcc}
\hline Generation Number & Chymosin activity $\mathbf{( U / g )}$ & Proteolysis activity (U/g) \\
\hline 1 & 516.12 & 1.2964 \\
2 & 510.06 & 1.1433 \\
3 & 511.36 & 1.1495 \\
4 & 509.27 & 1.1478 \\
5 & 510.52 & 1.1497 \\
\hline
\end{tabular}

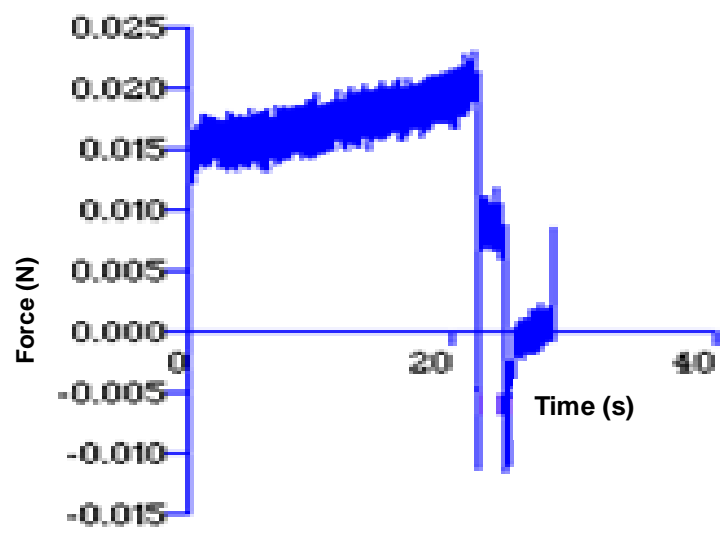

(a)

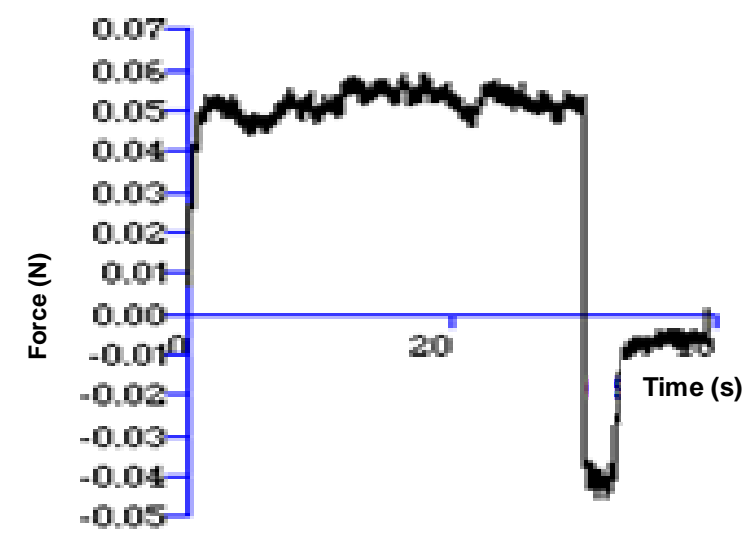

(b)

Figure 3. Texture analysis of the cheese by (a) rennet from original strain and (b) objective strain.

of this experiment. Its rennet activity has significantly improved, which has increased by $29.0 \%$ compared with the original strain.

\section{Genetic stability of the strain}

Some genetic types of mutant strain by artificial mutation were not stable, which prone to mutation or production fell back. So it was needed to verify its genetic stability. The data in Table 3 indicates that when BsB1.1 strain screened after mutagenesis inherits to the fifth generation, rennet activity is relatively stable, which shows that BsB1.1 strain has a favorable genetic stability and its characters can be stably inherited.

\section{Texture analysis of the cheese by rennet from original strain and objective strain}

From Figure 3, the texture analysis of the cheese by rennet from original strain and objective strain were obtained. The area blew the horizontal axis indicated the viscosity. It was found that the viscosity was had risen significantly through the microwave mutagenic, which enhanced the stability of the cheese effectively. So, it could be confirmed that the microwave mutagenic was an effective way to gain high-producing rennet strain from $B$. Subtilis.

\section{DISCUSSION}

Microwave mutagenesis studies have been widely carried out. Sasaki (1976-1978) had studied the mutant effect of the microwave on the mile bacteria, viruses, spores and lower eukaryotes yeast. It has been found that the production of Nisin from lactobacillus increased $42.13 \%$ than the original strain through microwave mutagenesis.

It is also shown from the experimental results that the best microwave mutagenic time of BsB1 strain is 4 min and the best mutagenic power is $450 \mathrm{~W}$. After mutagenesis, the rennet activity of BsB1.1 strain has increased by $29.0 \%$ and has a favorable genetic stability. Therefore, microwave mutagenesis is an effective way to cultivate viable $B$. subtilis strains with high-producing rennet. The mechanism of microwave mutagenic may be due to the following aspects: (1) Microwave is an electromagnetic wave, which can cause the rotation of the polar molecules such as water, proteins, nucleotides, fat, and carbohydrate. Especially when the water molecules were under microwave irradiation of 2450 $\mathrm{MHZ}$, it can rotate back and forth $24.5 \times 108$ times in $1 \mathrm{~s}$, 
which causing intense friction between the molecules. So it makes the hydrogen bonding and base stacking of intracellular DNA molecular chemical impaired, eventually cause the molecular structure of DNA change, and Lead to genetic variation. (2) The microwave has a strong penetrating effect, causing water molecules produce vigorous rotation, to change the cell wall permeability. It is easier to make intracellular enzyme secreted. (3) Strong thermal motion of molecules caused by the microwave was easy to cause inactivation of the enzyme, and make it easier for the intracellular enzyme secreted. (4) Microwave mutagenic effects of micro-organisms, in addition to the thermal effects, there are still non-thermal effects. But the mechanism of microwave mutagenic on the high-producing rennet strain from $B$. subtilis is still by further experiments to prove it.

From the experimental results it is indicated that the microwave treatment is a new technology for strain to improve the rennet. The technique is easy and safe to manipulate and the equipment requirement is low. It may be possible to apply this technology industrial microbial breeding engineering.

\section{Conflict of Interests}

The author(s) have not declared any conflict of interests.

\section{REFERENCES}

Chen YP, Cui Y, Ren ZY (2006). Correlation between growth development and biophoton emission of Isatis indigotica cotyledon exposed to microwave radiation. J. Infrared Millin Waves 25:275-278.

Hong SM, Park JK, Lee Y (2004). Mechanisms of microwave irradiation involved in the destruction of fecal coliforms frombiosolids. Water Res. 38(6):1615-1625.

Kirsschvink JL (1996) Microwave absorption by magnetite: a possible mechanism for coupling nonthermal level of radiation to biological systems. Bioelectromagnet J. 17:187-194.

Lamsa C, Bloebaum P (1990). Mutation and screening to increase chymosin yield in a genetically-engineered strain of Aspergillus awamori. J. Ind. Microbiol. 5:229-238.

Li JF, Zhang SQ, Shi SL (2009a). Screening of high IAA-producing rhizobium strain with antibiotic resistance using microwave utagenesis. J. Nucl. Agri. Sci. 23:981-985.
Li JF, Zhang SQ, Shi SL, Huo PH (2009b). Screening of dissolve phosphorus Rhizobium meliloti antibiotic-resistant strain using microwave mutagenesis. Atomic Energy Sci. Technol. 43:1071-1076.

Pan M, Zhou YJ (2008). Application of microwave technology in Saccharomyces Cerevisiae screening. China Brewing.11:78-80.

Selvakumar G, Kundu S, Gupta AD, Huche YS, Gupta HS (2008). Isolation and characterization of nonrhizobial plant growth promoting bacteria from nodules of Kudzu (Pueraria thunbergiana) and their effect on wheat seedling growth. Curr. Microbiol. 56:134-139.

Song L, Liu XF, Liu PW (2008).Screening of highly efficient H2producing aciduric anaerobic strain using microwave mutagenesis. Chin. J. Appl. Environ. Biol.14:427-443.

Woo IS, Rhee IK, Park HD (2000). Differential damage in bacterial cells by microwave radiation on the basis of cell wall structure. Appl. Environ. Microbiol. 66:2243-224.

Xu W, Wang P, Zhang X (2009). Breeding and characterization of $\mathrm{LI}$ actic acid high-yielding strain by microwave mutation. J. Tianjin University. 42:544-548. 